\title{
Dynamical Planning Simulation of Quadruped Robot Based on Force Control Model
}

\author{
Bingqiang Yan ${ }^{1,2}$ and Xinjian $\mathrm{Li}^{1}$ \\ 1. School of Mechatronical Engineering, Beijing Institute of Technology,5 South \\ Zhongguancun Street, Haidian District, Beijing ,100081,China \\ 2. Hebei College of Industry and Technology, Shijiazhuang, 050091, China
}

\begin{abstract}
The quadruped robot can play an important role in many complex environments, in order to improve design efficiency of it, the dynamical planning simulation of it based on force control model is studied in depth. Firstly, the mechanism analysis of quadruped robot is analyzed, and the corresponding dynamical models of it are studied in depth. Secondly, the dynamical planning algorithm of quadruped robot based on force controlling module is analyzed. Finally, the dynamical planning simulation of quadruped robot is carried out using a quadruped robot as example, results show that the force control model can improve the stability of quadruped robot effectively.
\end{abstract}

Keywords: quadruped robot; mechanism analysis; dynamical model; dynamical planning simulation

\section{Introduction}

With development of science and technology, the quadruped robot can play an important role in many complex environments, such as rugged mountain road, earthquake fire scene, during the procession of designing the quadruped robot, the procedure "design-manufacture-test" should be carried out repeatedly, finally the design can be confirmed. In order to reduce the manufacturing cost and period, the simulation technology should be applied in verifying the rationality of gait planning and structure design, and simulation results can offer effective basis for design the material object, the design efficiency can be improved. The quadruped robot has multiple branched kinematic mechanisms, which has time-variant motion topology mechanism, and it also is a kind of redundant drive system. Every leg of quadruped robot with full range maneuvering performance has at least three driving joints, therefore the quadruped robot has twelve driving joints, and the mechanism diagram of quadruped robot is shown in Figure 1 [1]. 


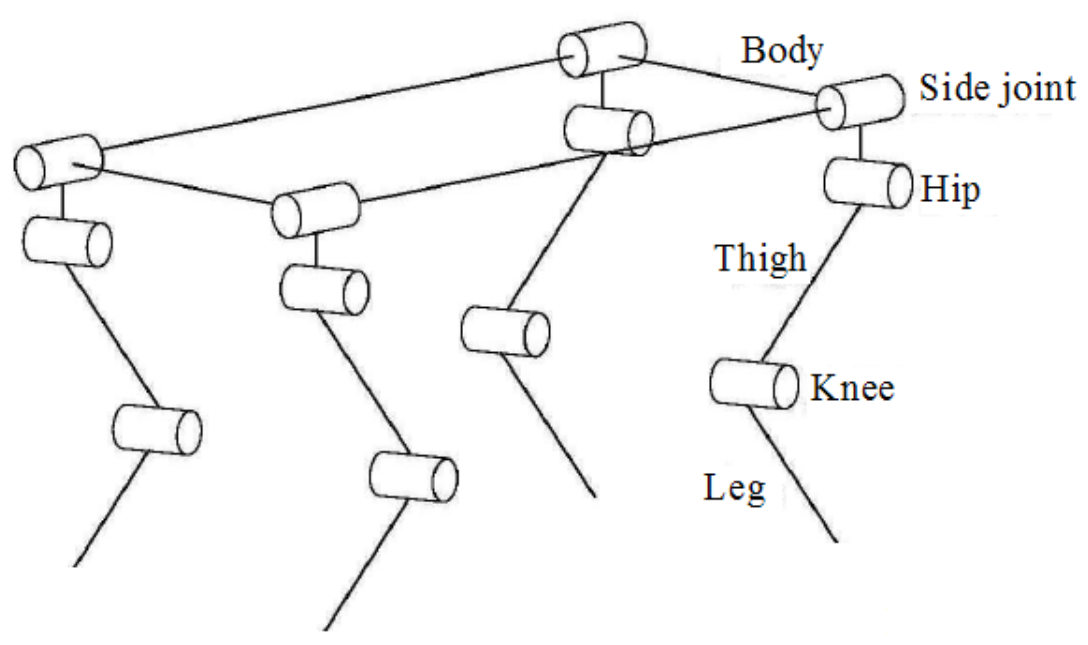

Figure 1. Structure Diagram of Quadruped Robot

The number of driving joints is far more than that of motion freedom, therefore the dynamical analysis of quadruped robot is very complex, therefore an advanced controlling technology should be applied and the force controlling model is used in this research.

\section{Mechanism Analysis of Quadruped Robot}

According to the hierarchy of quadruped robot, the organization level and module of it can be divided, which is shown in Figure 2.

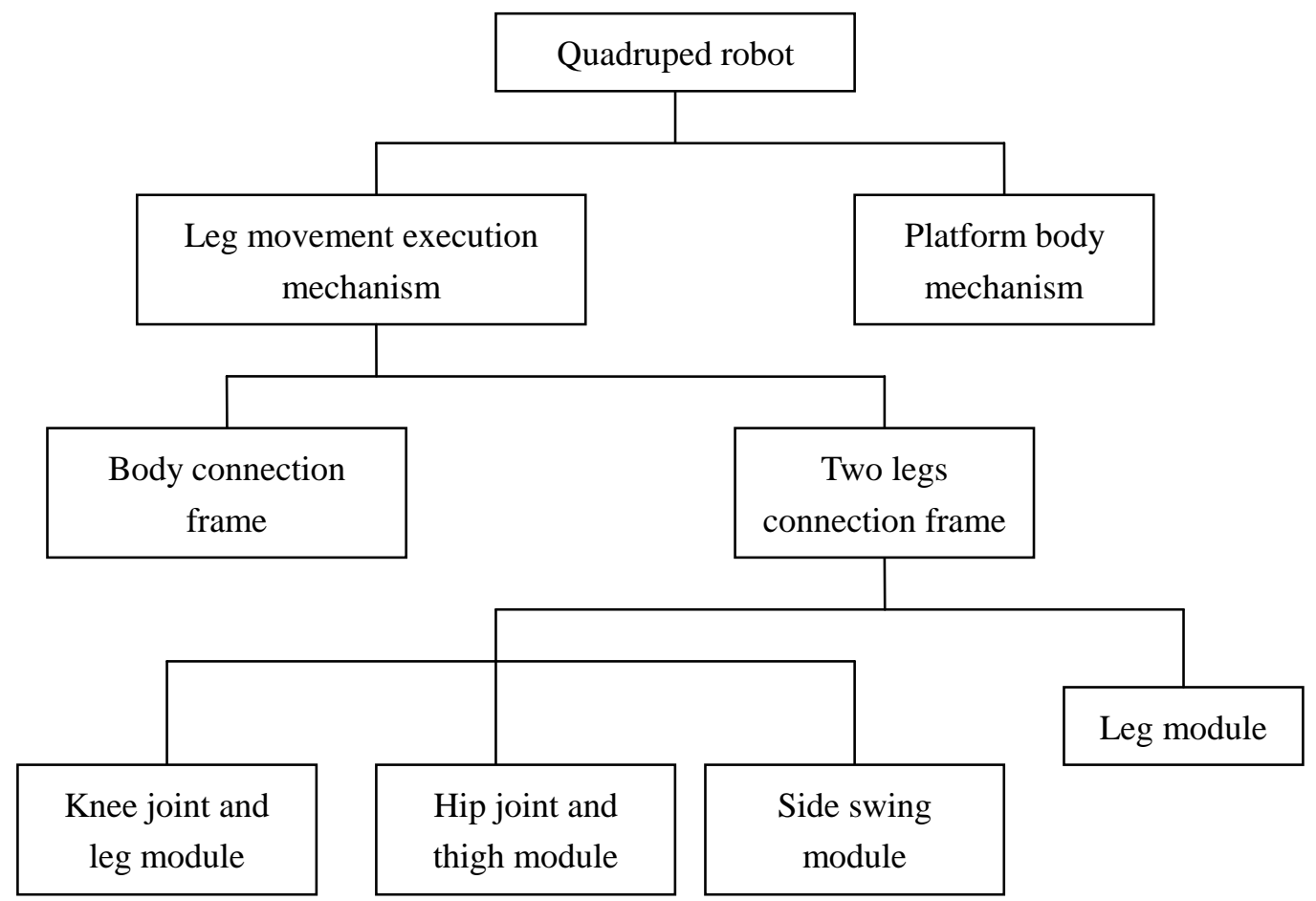

Figure 2. Module Division Diagram of Quadruped Robot

All components of quadruped robot are organized and planned based on "part-component-parts-accessory" four levels, where the side swing module, hip joint and 
thigh module, knee joint and leg module are limits, the bottom is the part layer should be designed in depth, the leg module and two legs module have independent function, which can be made up of an part layer.

The leg of quadruped robot can be simplified as the kinematic mechanism, which is shown in Figure 3. The length of leg is defined by $l$, which is the distance between hip joint and foot joint of robot, the angle of leg is defined by $\theta_{v}$, which is the intersection angle between the connecting line of hip joint and foot joint and Ab/Ad joint axis line, the following theoretical models are listed as follows [2]:

$$
\begin{gathered}
l=\sqrt{x_{A i}^{2}+y_{A i}^{2}} \\
\theta_{v}=\pi-\arctan \left(\frac{x_{A i}}{y_{A i}}\right)
\end{gathered}
$$

where $x_{A i}=l_{i 2}+l_{i 1} \cos \theta_{i a}-\left(l_{i 5}+l_{i 6}\right) \cos \theta_{3}, \quad y_{A i}=l_{i 1} \cos \theta_{i a}-\left(l_{i 5}+l_{i 6}\right) \sin \theta_{3}$.

The velocity model of quadruped robot is expressed as follows:

$$
\vec{V}_{A i}=J \dot{\vec{q}}
$$

where $\vec{V}_{A i}$ denotes the velocity vector of point $A_{i}, \vec{V}_{A i}=\left[\dot{x}_{A i}, \dot{y}_{A i}, \dot{\theta}_{3}\right]^{T} ; \dot{\vec{q}}$ is generalized velocity vector, $\dot{\vec{q}}=\left[\dot{\theta}_{i b}, \dot{\theta}_{i a}\right] ; J$ is the velocity Jacobin matrix of point $A_{i}$ to driving joint.

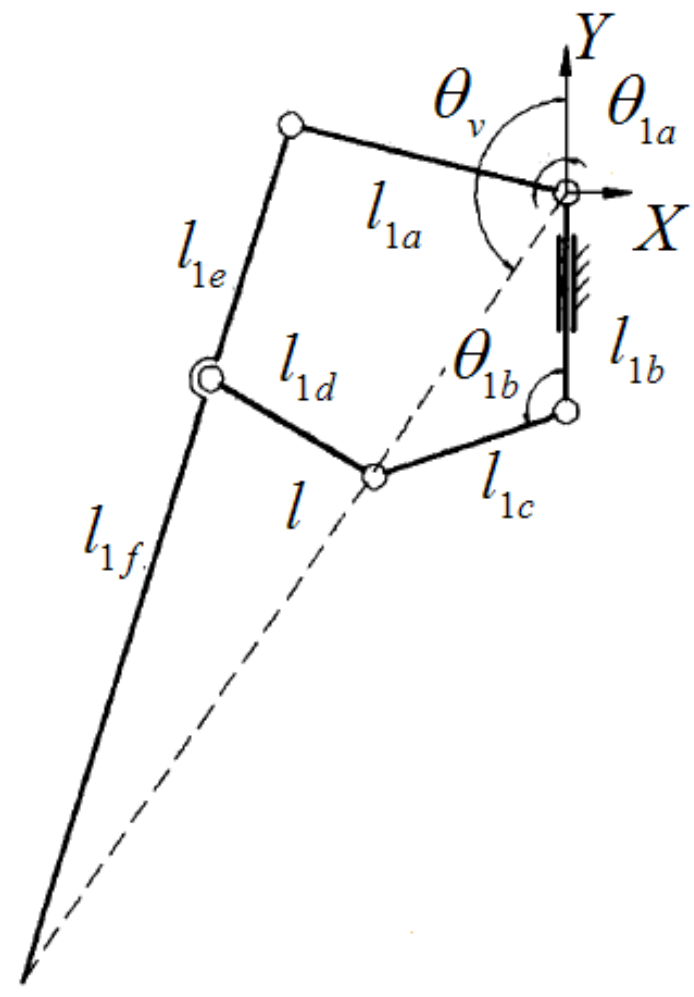

Figure 3. Simplified Diagram of Leg Kinematic Mechanism

During the procession of controlling the quadruped robot, the velocity inverse solution should be considered, and the corresponding solution equation is expressed as follows [3]:

$$
\dot{\vec{q}}=J^{-1} \vec{V}_{A i}
$$

where $J^{-1}$ is the generalized inverse of Jacobin matrix. 
The relationship between the acceleration vector of point $A_{i}$ for robot and that of active joint can be expressed as follows:

$$
\vec{V}_{A i}=\dot{J} \dot{q}+J \ddot{q}
$$

where $\dot{J}$ is the derivative of Jacobin matrix to time.

The acceleration model of inverse kinematics problem for quadruped robot can be expressed as follows [4]:

$$
\ddot{q}=J^{-1}\left(\dot{\vec{V}}_{A i}-\dot{J} \dot{q}\right)
$$

When the acceleration of active joint is known, the acceleration of reference point $A_{i}$ can be obtained according to expression (4). When the acceleration of reference point $A_{i}$ is known, the acceleration of active joint can be solved based on expression (5).

\section{Dynamical Planning Algorithm of Quadruped Robot Based On Force Controlling Module}

The force controlling module of quadruped robot can make the robot move according to the specified trace based on driving force torque of every joint of quadruped robot.

The torque of quadruped robot can be solved based on iteration Newton-Euler algorithm, the dynamical equation of a leg link $i$ is expressed as follows [5]:

$$
\begin{aligned}
& f_{\sum_{i}}=m_{i} \ddot{c}_{i} \\
& \tau_{i}^{(c)}=I_{i} \dot{\omega}_{i}+\omega_{i} I_{i} \omega_{i}
\end{aligned}
$$

where $f_{\sum_{i}}$ is the resultant external force acting on the centre of mass, $\tau_{i}^{(c)}$ is the external torque around the centre of mass.

$$
\dot{v}_{i}=\ddot{c}_{i}+\dot{\omega}_{i} c_{i}-\omega_{i} \times \dot{c}_{i}
$$

Then the following expression can be obtained:

$$
f_{\sum_{i}}=m_{i}\left(v_{i}-c_{i} \times \omega_{i}+\omega_{i} \times\left(v_{i}+\omega_{i} \times c_{i}\right)\right)
$$

The resultant moment in the global coordinate system formed by $f$ and $\tau_{i}^{(c)}$ can be expressed as follows:

$$
\tau_{\Sigma i}=\tau_{i}^{(c)}+c f=I_{i} \omega_{i}+\omega_{i} I_{i} \omega_{i}+m c \cdot\left(v_{i}-c_{i} \times \omega_{i}+\omega_{i}\left(v_{i}+\omega_{i} \times c_{i}\right)\right)
$$

The matrix representation is arranged based on expressions (9) and (10), which is expressed as follows [6]:

$$
\left[\begin{array}{l}
f_{\Sigma i} \\
\tau_{\Sigma i}
\end{array}\right]=I_{i}^{S} \ddot{X}_{i}+\dot{X}_{i} \times I_{i}^{S} \dot{X}_{i}
$$

where $I_{i}^{S}$ is the $6 \times 6$ symmetrical matrix, which is named as space inertia matrix, which is calculated by the following expression:

$$
I_{i}^{S}=\left[\begin{array}{cc}
m_{i} E & m_{i} \hat{c}_{i}^{T} \\
m_{i} \hat{c}_{i} & m_{i} \hat{c}_{i} \hat{c}_{i}^{T}+I
\end{array}\right]
$$

For the leg link system of quadruped robot the resultant force and torque acting on the leg link can be expressed as follows [7]:

$$
\left[\begin{array}{l}
f_{\Sigma i} \\
\tau_{\Sigma i}
\end{array}\right]=\left[\begin{array}{l}
f_{i} \\
\tau_{i}
\end{array}\right]+\left[\begin{array}{l}
f_{i}^{E} \\
\tau_{I}^{E}
\end{array}\right]-\left[\begin{array}{l}
f_{i+1} \\
\tau_{i+1}
\end{array}\right]
$$

where $f_{i}$ and $\tau_{i}$ are the force and moment acting on leg link $i ; f_{i}^{E}$ and $\tau_{I}^{E}$ are 
the force and moment coming from outer environment. $f_{i+1}$ and $\tau_{i+1}$ reaction force and moment.

The recursive equation of force and moment can be obtained through substituting expression (13) into expression (11):

$$
\left[\begin{array}{c}
f_{i} \\
\tau_{i}
\end{array}\right]=I_{i}^{S} \ddot{X}_{i}+\dot{X}_{i} \times I_{i}^{S} \dot{X}_{i}-\left[\begin{array}{c}
f_{i}^{E} \\
\tau_{i}^{E}
\end{array}\right]+\left[\begin{array}{c}
f_{i+1} \\
\tau_{i+1}
\end{array}\right]
$$

If the link $i$ is the end link of quadruped robot leg, the value of $f_{i+1}$ and $\tau_{i+1}$ can be set as zero when $f_{i}$ and $\tau_{i}$ are calculated. When the leg of link belongs to kicking leg, then $f_{i}^{E}$ and $\tau_{i}^{E}$ are gravity and gravity moment respectively. Otherwise, when the leg of link is supporting leg, then $f_{i}^{E}$ and $\tau_{i}^{E}$ conclude gravity item and contacting force item between the link and ground.

The torque acting on the joint axis can be expressed as follows [8]:

$$
u_{i}=s_{i}^{T}\left[f_{i}, \tau_{i}\right]
$$

When the driving force of every joint for the quadruped robot is calculated, the control with quick speed and high precision of it can be achieved.

\section{Dynamical Simulation of Quadruped Robot}

In order to verify the effectiveness of force controlling model, the corresponding simulation analysis is carried out, the limb structure of quadruped robot is simplified, every leg has three joints, where the hip joint has two rotating freedom, and the knee and ankle joints have a rotating freedom respectively. The height of quadruped robot is $847 \mathrm{~mm}$, the length of quadruped robot is $582 \mathrm{~mm}$, and the width is $284 \mathrm{~mm}$, the length of hip joint is $88 \mathrm{~mm}$, the length of thigh joint is $172 \mathrm{~mm}$, the length of calf is $194 \mathrm{~mm}$.

The displacement and velocity of quadruped robot in $\mathrm{X}, \mathrm{Y}$ and $\mathrm{Z}$ directions are shown in Figures 4-6. The $X$ direction of quadruped robot is advancing direction. As seen from Figure 4, the body of quadruped robot moves with low velocity at the beginning, and then accelerates gradually. Finally, the quadruped robot keeps the movement with stable speed.

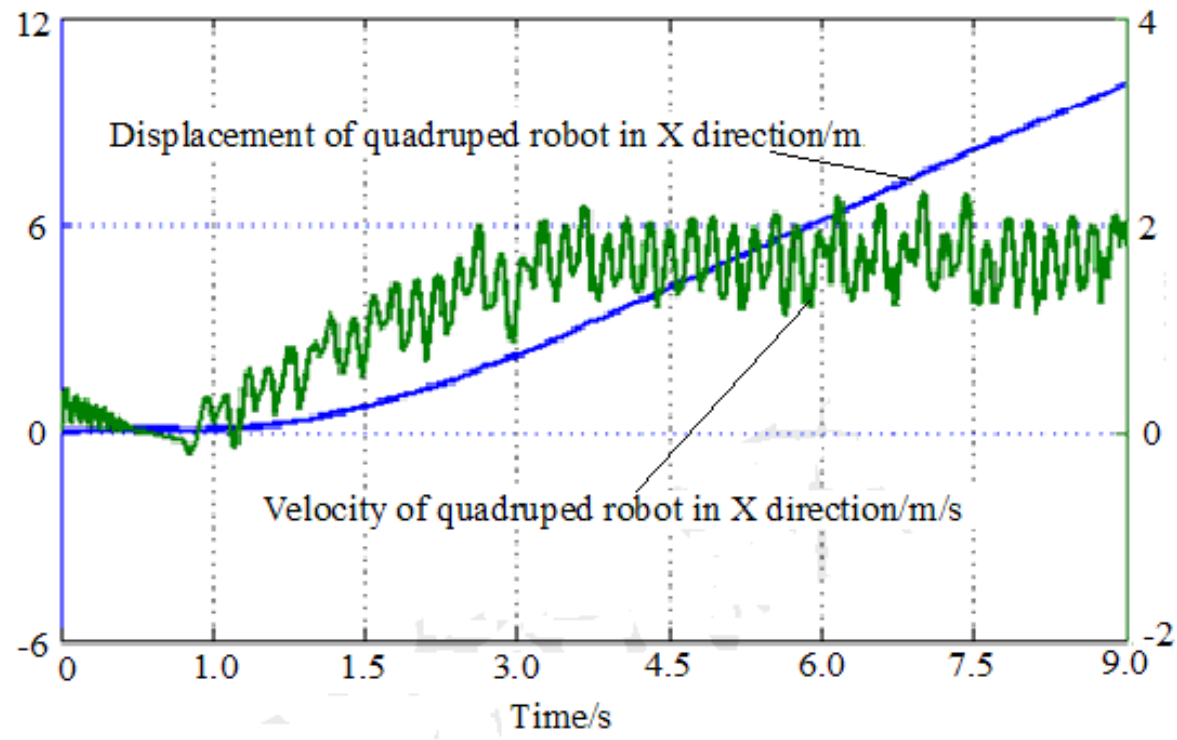

Figure 4. Displacement and Velocity of Quadruped Robot in X Direction

Figure 5 shows the displacement and velocity in $\mathrm{Y}$ direction. The barycenter 
displacement of quadruped robot changes in the range of $\pm 3 \mathrm{~mm}$, therefore the robot can move stably, and there is no jumping and falling phenomenon.

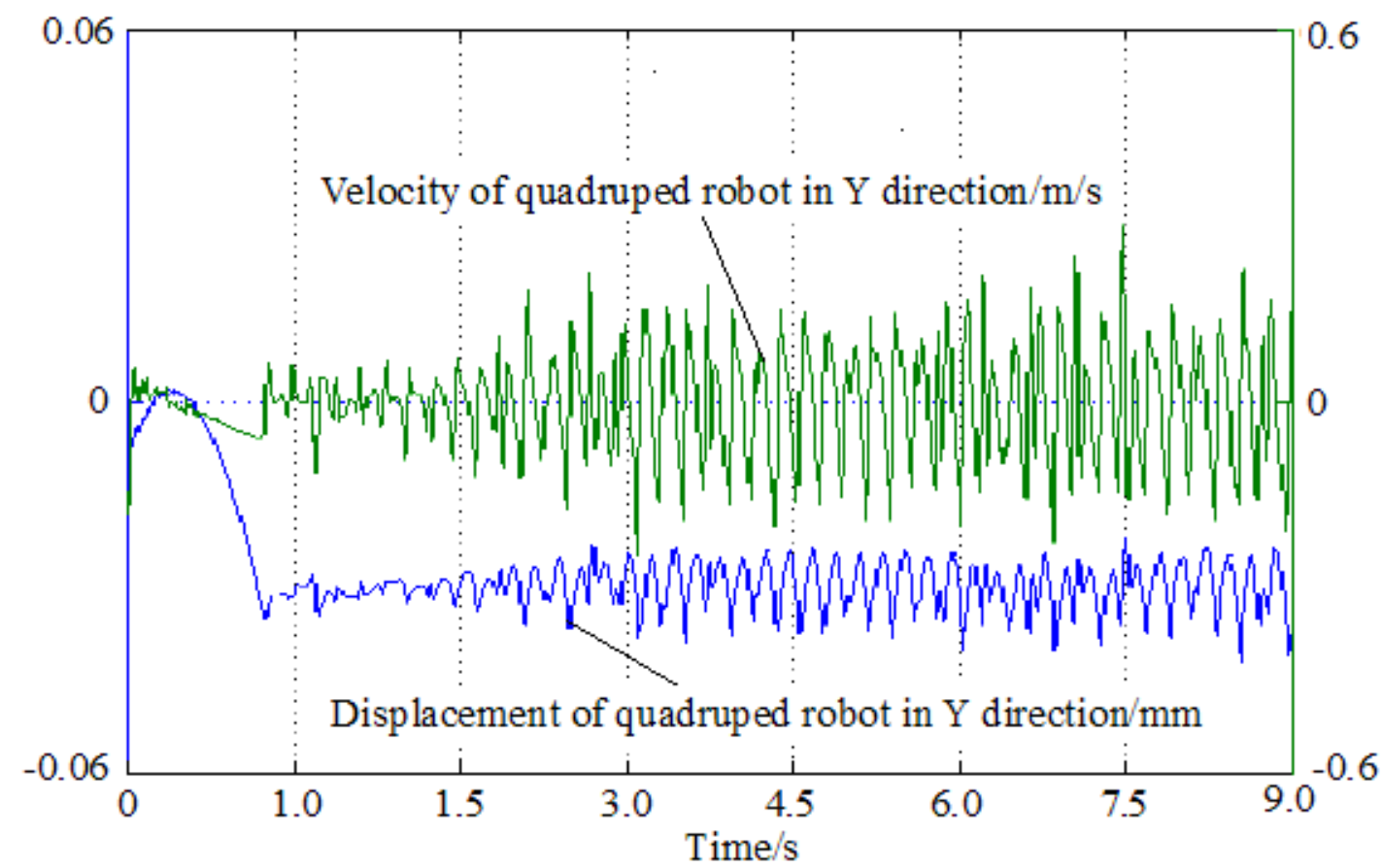

Figure 5. Displacement and Velocity of Quadruped Robot in Y Direction

Figure 6 shows the horizontal moving displacement and velocity of quadruped robot, because the quadruped robot has no yaw control, there is about $2.4 \mathrm{~m}$ displacement in horizontal direction at $9 \mathrm{~s}$, this phenomenon is caused by controlling the landing point of kicking leg when the position of the body is regulated.

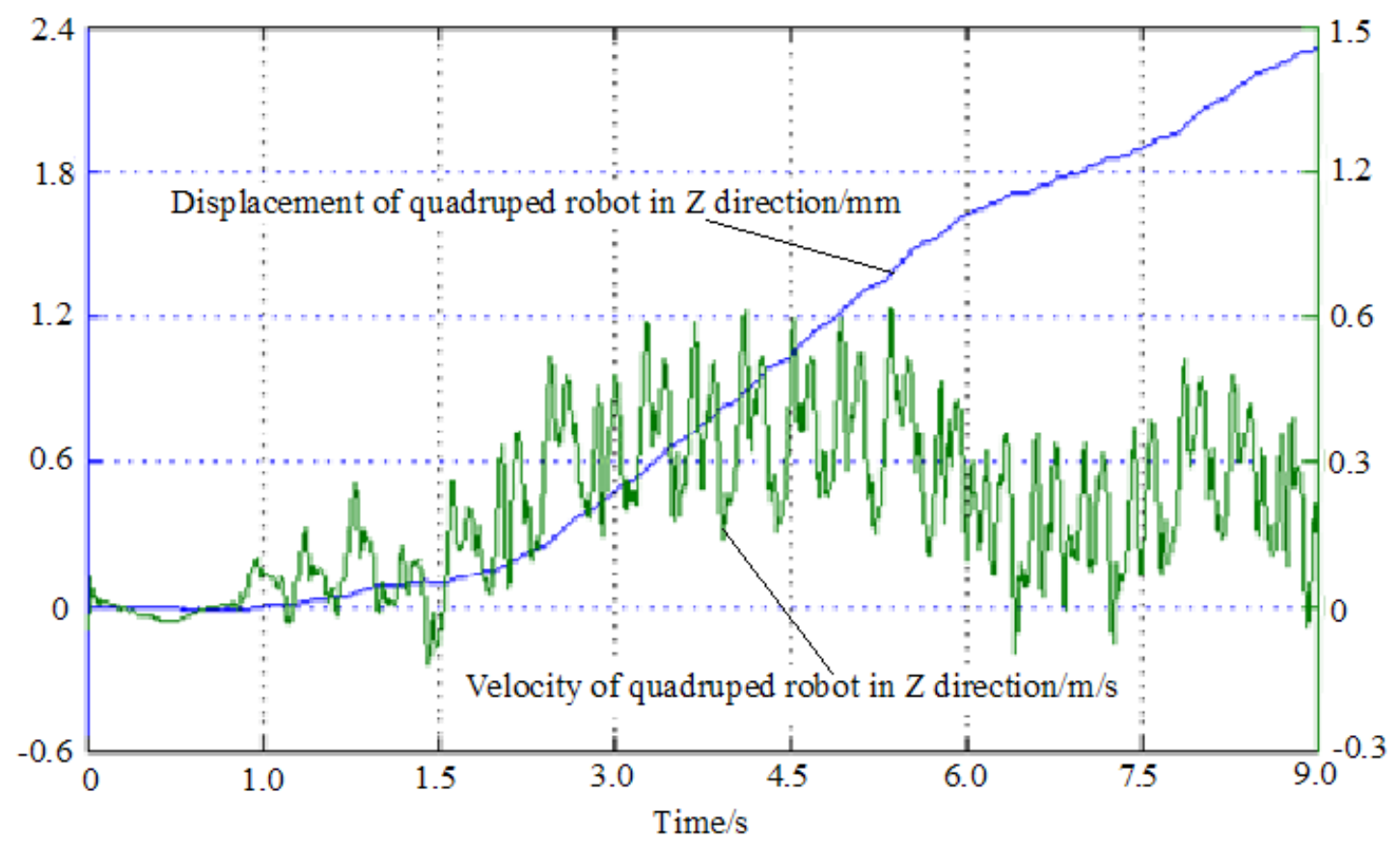

Figure 6. Displacement and Velocity of Quadruped Robot in Z Direction 


\section{Conclusions}

The force controlling model is applied in the dynamical planning of quadruped robot, and the corresponding dynamical simulation of quadruped robot is carried out. Simulation results show that the quadruped robot can move according to the specified walk and route, the body of quadruped robot moves with low velocity at the beginning, and then accelerates gradually, the bar center displacement of quadruped robot changes in the range of $\pm 3 \mathrm{~mm}$, the quadruped robot has no yaw control, there is about $2.4 \mathrm{~m}$ displacement in horizontal direction at $9 \mathrm{~s}$, and movement of it is stable, therefore the force controlling model is an effective method for controlling the walk of quadruped robot, the stability of it can be improved effectively.

\section{References}

[1] P. T. Doan, H. D. Vo, H. K. Kim and S. B. Kim, "A new approach for development of quadruped robot based on biological concepts", International Journal of Precision Engineering and Manufacturing, vol.11, no.4, (2010), pp.559-568.

[2] B. Wang, R. Hu, X. Zhang and C. Huai, "Gait planning and intelligent control for a quadruped robot", Journal of Control Theory and Applications, vol.7, no. 2, (2009), pp.207-211.

[3] S. J. Sugumar and R. Jayaparvathy, "Design of a quadruped robot for human-elephant conflict mitigation", Artificial Life and Robotics, vol.18, no.3-4, (2013), pp.204-211.

[4] J.-M. Yin, H.-K. Li and Z.-D. Dai, "Quadruped robot harmonious control based on RBF-Q learning", Application Research of Computers, vol.30, no.8, (2013), pp.2349-2352. (In Chinese)

[5] K.-L. Zhou, X.-L. Zhang and J.-G. Gong, "Transition control of a quadruped robot from quadruped to biped locomotion”, Mechanical \& Electrical Engineering Magazine, vol.30, no.1, (2013), pp.15-20. (In Chinese)

[6] S.-W. Lee, O-H. Kwon and K.-H. Rim, "Fuzzy Controller Design for Quadruped Robot Posture Control", International Journal of Digital Content Technology and its Applications, vol.7, no.12, (2013), pp.228-233.

[7] D. T. Tran, I. M. Koo and Y. H. Lee, "Motion control of a quadruped robot in unknown rough terrain using 3D spring damper leg model", International Journal of Control, Automation, and Systems, vol.12, no.2, (2014), pp.372-382.

[8] B. Chen, Z.-C. Pei and C. Chen, "Bionic Motion Control Research of Hydraulic Quadruped Robot", Journal of System Simulation, vol.25, no.8, (2013), pp.1746-1750. (In Chinese) 
International Journal of Hybrid Information Technology

Vol. 10, No.2 (2017) 